\title{
Gesundheitswesen:
}

\section{vom 1. April zu künftigen Entwicklungen}

Der 1. April mit seinen kantonalen Kundgebungen der Hausärztinnen und Hausärzte war ein voller Erfolg! Die Zahl der Teilnehmenden an den verschiedenen Demonstrationen war im Allgemeinen sehr hoch (15000 schweizweit), aber vor allem kam die Botschaft in der Öffentlichkeit an: Es geht um nichts Geringeres als die Zukunft der medizinischen Versorgung des Landes, um den Fortbestand der Hausarztmedizin, und nicht nur um ein paar Franken bei Laboranalysen! Zu erwähnen ist dabei auch die grosse Solidarität der Schweizer Ärzteschaft, die sich weder durch das Laborthema noch durch die Frage der Einkommensverteilung spalten lässt.

Zusammen mit der Veröffentlichung der Einkommensverhältnisse der Ärzteschaft vor einigen Wochen [1] sowie mit aufkommenden Gerüchten über einen massiven Prämienschub bei den Krankenkassen hat der Aktionstag der SGAM die Politik aufgerüttelt, eine ganze Reihe neuer Ideen hervorgebracht und letztlich die Karten neu verteilt.

Vielleicht ist nun endlich die Zeit gekommen, veraltete Vorstellungen über Bord zu werfen und zu neuen Ufern aufzubrechen, die Beziehungen sowie die Methoden des Dialogs und der Absprache zwischen den Akteuren dieses kriselnden Systems ganz im Sinne der letzten öffentlichen Stellungnahmen der FMH zu überdenken. Wir wollen den Dialog auf jeden Fall in dieser Richtung fortsetzen.

Leider sind einige der in jüngster Zeit geäusserten Ideen nutzlose Alibiübungen, mit denen die seit 15 Jahren ver- sind viel zu wenig zahlreich, um irgendetwas Nützliches zu einem möglichen «gemeinsamen Topf» beitragen zu können.

- Schliesslich muss anerkannt werden, dass die in den vergangenen Wochen im Zentrum der Medienberichterstattung stehenden Facharzteinkommen hauptsächlich aus Leistungen ausserhalb der Grundversicherung bestehen, sodass eine «TARMED-Neuverhandlung» daran nichts ändern würde.

Allein die Idee, das Problem den Ärzten zuschieben zu wollen, ist grundlegend falsch. Wer vorgibt, das Schweizer Gesundheitswesen ohne mehr Mittel ausbauen zu können, gibt sich einer Illusion hin und täuscht die Bevölkerung.

Nein, es müssen andere Lösungen gefunden werden: mittelfristig zur Sicherung einer angemessenen Versorgung in der ganzen Schweiz und kurzfristig zur Bekämpfung des bevorstehenden Prämienanstiegs - beiden Entwicklungen wollen wir nicht untätig zusehen. In nächster Zeit stehen lebhafte Diskussionen an. Zu den Themen werden sicherlich die Medikamente und Franchisen gehören, aber auch andere Ideen werden wieder auf den Tisch kommen, beispielsweise im Hinblick auf eine Nachfolgeregelung zum Zulassungsstopp.

Auch das Thema Managed Care dürfte wieder aktuell werden, aber Managed Care auf freiwilliger Basis mit ausgewogenen Anreizen, Qualitätsgarantien und wahr-

\section{Die Idee, das Problem den Ärzten zuschieben zu wollen, ist falsch. Wer vorgibt, das Schweizer Gesundheitswesen ohne mehr Mittel ausbauen zu können, gibt sich einer Illusion hin}

fahrene Situation nur verlängert wird. Besonders bedauerlich ist, dass einige Politiker es immer noch für hilfreich erachten, sich mit solchen Aussagen zu profilieren.

Ich denke dabei an die Idee einer Einkommensumverteilung innerhalb der Ärzteschaft und sage dazu klar: Die FMH akzeptiert es nicht, dass gewisse ausgebrannte und ideenlose Politiker die Folgen ihrer Regierungsunfähigkeit auf die Ärzteschaft überwälzen. Die Argumente, die zeigen, wie absurd dieser Vorschlag ist, sind bekannt, weshalb ich sie nur kurz erwähnen möchte:

- Das zur Aufwertung der Hausarztmedizin notwendige Finanzvolumen existiert innerhalb der Ärzteschaft schlichtweg nicht.

- Es gibt Tausende von Fachärzten, die noch weniger verdienen als die Allgemeinpraktiker, z.B. die Psychiater.

- Die Fachärzte mit anständigem Einkommen (aber nicht mehr, als jeder andere freie Beruf haben sollte) scheinlich doch einer finanziellen Verantwortung. Alle Initiativen in dieser Richtung werden wir sicherlich unterstützen und uns daran beteiligen; diese werden wir selbstverständlich je nach ihrer Entwicklung an dieser Stelle wieder aufgreifen.

Kurz: Es ist zu hoffen, dass wir unter Partnern des Gesundheitswesens rasch einige gemeinsame Nenner definieren, die zu umsetzbaren Vorschlägen führen - nicht nur, weil die Zeit drängt, sondern weil die Verhältnisse nun endlich günstig scheinen: Das sollten wir nutzen, um konstruktiv und innovativ weiterzuarbeiten, auch auf der Ebene der Politik!

Dr. med. Jacques de Haller, Präsident der FMH

1 Hasler N, Reichert M. Einkommensverhältnisse der freien Ärzteschaft der Schweiz in den Jahren 2005 (neu) und 2004 (Re-Evaluation). Schweiz Ärztezeitung. 2009;90(11):409-19. 